\title{
Collaborative Information Agents on the World Wide Web
}

\author{
James R. Chen*, Nathalie Mathé ${ }^{*} \&$ Shawn Wolfe ${ }^{\dagger}$ \\ NASA Ames Research Center \\ Moffett Field, CA 94035-1000 \\ \{jchen, mathe, shawn\}@ptolemy.arc.nasa.gov
}

\begin{abstract}
In this paper, we present DIAMS, a system of distributed, collaborative information agents which help users access, collect, organize and exchange information on the World Wide Web. Personal agents provide their owners dynamic displays of well organized information collections, as well as friendly information management utilities. Personal agents exchange information with one another. They also work with other types of information agents such as matchmakers and knowledge experts to facilitate collaboration and communication.
\end{abstract}

KEYWORDS: intelligent agents, information access, collaborative system, knowledge-base, world wide web

\section{INTRODUCTION}

Evolution of the World Wide Web has greatly enhanced the wealth of information access on the Internet. The task of finding the most relevant information at any time given particular user needs, however, has correspondingly gained much more complexity. With the abundance of information resources, organizing and managing personal or public URL collections has also become more difficult. While existing WWW search engines and centralized catalogs (eg. InfoSeek, OpenText, Yahoo, etc.) provide relevant information and useful services, they are typically not customizable to match individual needs. Various intelligent systems have been developed to support features which enhance information access, integrate different resources, improve search precisions, etc.. Few systems, however, make good use of the intelligence of individual users, and the possible collaborations between them. We propose DIAMS, a system of distributed, collaborative information agents which help users access, collect, organize and exchange information on the World Wide Web. DIAMS supports users with friendly tools for the intelligent organization of their information collections. Moreover, it provides automated communication and collaboration between different individuals and/or groups, to facilitate information sharing.

"contracted through Recom Technologies

${ }^{\dagger}$ contracted through Caelum Research

\section{PERSONAL INFORMATION AGENTS}

DIAMS incorporates several different types of information agents. Among them, personal agents are the ones that work most directly with users to help support the presentation, organization and management of user information collections. A personal agent gives its user easy and effective access to its collection contents. It also provides its owner with friendly tools to manipulate and update the contents.

Like most Web browsers' bookmarking facilities, a DIAMS agent provides flexible hierarchical displays of any subsets of its information contents. It accommodates multiple parents, nested categories, aliases and loops in the structure, thereby supporting dynamic organizations of the same or similar subcollections when needed. DIAMS combines friendly graphical access with powerful query search. A category in DIAMS can be presented as a folder in a hierarchy, or used as an index in a query. Users can create and manage their own categories, URL contents and structures for easy and effective future access.

A user can browse or query other users' collections through his/her own personal agent. A personal agent can store external links to other agents, or to any parts of their collections. These external links can be manipulated and displayed just like other categories or URLs. Users can also import copies of selected sub-collections from other agents.

A personal agent can optionally maintain a miniature knowledgebase which provides the facilities to encode, display and utilize semantic relations, such as isa, partof, related, etc., among categories. This knowledge-base supports more sophisticated information organization and display.

User interface design and front end capabilities of prototype personal agents in DIAMS have been introduced in [?].

\section{COMMUNICATION AND COLLABORATION}

Personal agents collaborate closely with one another. They also work with other types of information agents in DIAMS which provide different kinds of services. Some of the functionalities of these agents and the relations among them are depicted in figure 1.

\section{Collaboration between Personal Agents}

Personal agents exchange information with one another. When a user query is directed to an external agent, the user's agent not only sends the query information, but also sends with the query its own answers, i.e., its information contents related to that query. The receiver agent, aside from replying to the query, has various options to handle the extra information that 


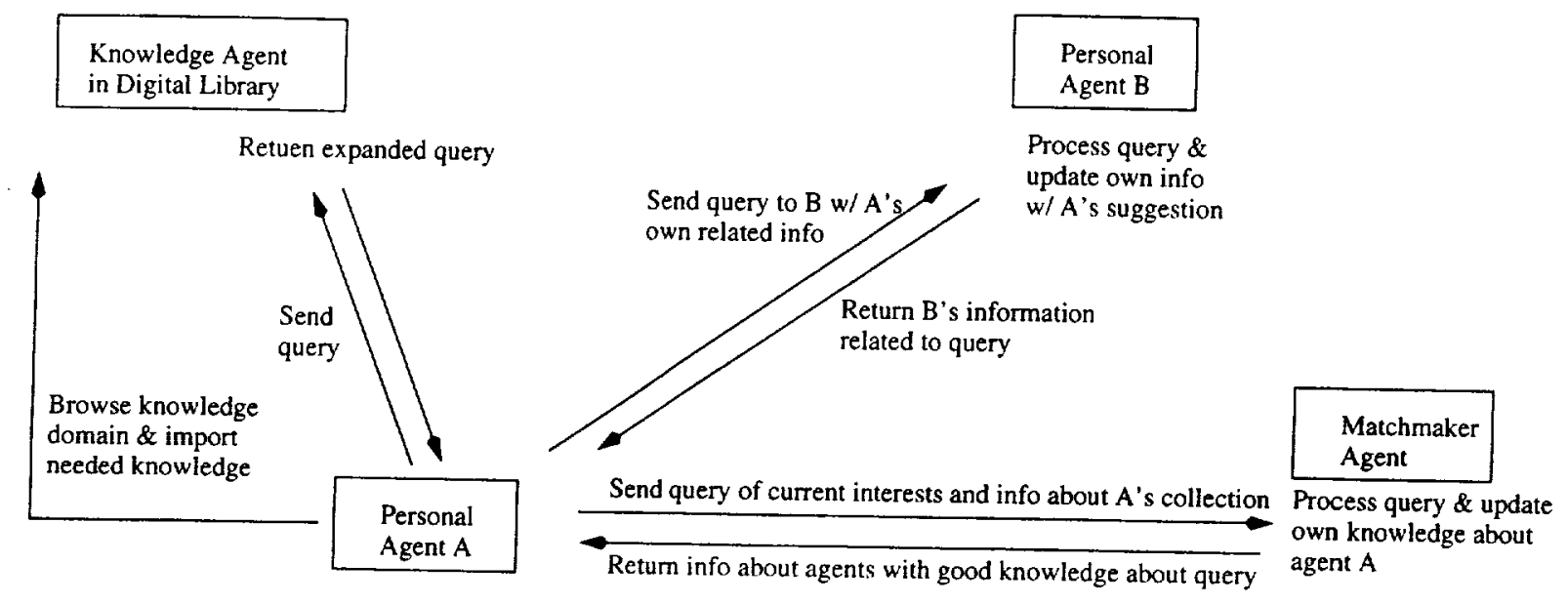

Figure 1: Collaboration and Communication between Agents

came with the query. It can digest the new information and place that into its own collection, or it can just ignore the new information. It can also keep the information in a temporary space and leave the handling decision to its owner.

Since categories of a personal agent are typically named by its owner, they are often not known to other agents. To facilitate communication between agents, each personal agent maintains a set of probabilistically most useful keywords extracted from its collection of documents, and the relations between these keywords and the categories. These keywords provide more commonalities between personal agents and thereby enhance communication between them. Specifically, they are used to translate queries by measuring similarities between categories of different users.

\section{Matchmaker Agents}

A matchmaker agent maintains information about personal agents. Its internal configuration is similar to that of a personal agent. However, instead of providing URL information associated with a query, a matchmaker provides the inquirer with links pointing to other agents which may carry information most relevant to the query.

When communicating with a matchmaker, the inquiring personal agent brings along with the query a set of categories and keywords representing its current collection and main interests. A matchmaker agent hence both receives and provides information about the collections of visiting personal agents.

\section{Knowledge Agents}

A personal agent can maintain its own miniature knowledgebase to help organize and manage its collection. Larger size knowledge-base with more complex semantic relations can be stored in a knowledge agent in DIAMS. Knowledge agents can carry expertise in various special domains. They are usually customized by domain experts, but can also learn new information from their visitors. Personal agents can import knowledge structures and/or commonly used categories from these knowledge agents. Knowledge agents are also used to expand queries into more elaborate descriptions, for better communication between personal agents.

\section{Other Utility Agents}

An important aspect of the DIAMS design is the capability of agents to not only communicate and interact with internal agents within the system, but also to make use of various existing systems and technologies on the web. Current version of DIAMS can import and export Netscape bookmarks. Many other important utilities such as access to dictionaries, thesauri, or other knowledge-bases, need to be developed. Interactions with major external systems will be coordinated by different utility agents, customized for their special purposes.

\section{EVOLUTION OF INFORMATION AGENTS}

We have introduced different types of agents in a DIAMS system. Each agent learns from its owner, as well as from other agents, to update its information and knowledge. Even largely customized knowledge agents need frequent updates to keep up with the changing world and growing technologies. The entire system of agents operate under a process of evolution. Each agent has limited capacity, and continually replaces unused information with new information. Useful agents learn more information from visitors and develop into experts. Agents with little interaction with others become less useful to the matchmakers and may disappear from the system. DIAMS agents thus evolve in their own specialties and contribute together to the World Wide Web.

\section{REFERENCES}

1. N. Mathé and J.R. Chen. Organizing and sharing information on the world-wide web using a multi-agent system. In Proc. of ED-MEDIA'98, Conference on Educational Multimedia and Hypermedia, 1998. 